\title{
Review Article \\ Testing General Relativistic Predictions with the LAGEOS Satellites
}

\author{
Roberto Peron ${ }^{1,2}$ \\ ${ }^{1}$ Istituto di Astrofisica e Planetologia Spaziali (IAPS-INAF), Via del Fosso del Cavaliere 100, 00133 Roma, Italy \\ ${ }^{2}$ Istituto Nazionale di Fisica Nucleare (INFN), Sezione di Roma Tor Vergata, Via della Ricerca Scientifica 1, 00133 Roma, Italy \\ Correspondence should be addressed to Roberto Peron; roberto.peron@iaps.inaf.it
}

Received 8 December 2013; Accepted 12 March 2014; Published 22 May 2014

Academic Editor: Douglas Singleton

Copyright (C) 2014 Roberto Peron. This is an open access article distributed under the Creative Commons Attribution License, which permits unrestricted use, distribution, and reproduction in any medium, provided the original work is properly cited. The publication of this article was funded by SCOAP $^{3}$.

\begin{abstract}
The spacetime around Earth is a good environment in order to perform tests of gravitational theories. According to Einstein's view of gravitational phenomena, the Earth mass-energy content curves the surrounding spacetime in a peculiar way. This (relatively) quiet dynamical environment enables a good reconstruction of geodetic satellites (test masses) orbit, provided that high-quality tracking data are available. This is the case of the LAGEOS satellites, built and launched mainly for geodetic and geodynamical purposes, but equally good for fundamental physics studies. A review of these studies is presented, focusing on data, models, and analysis strategies. Some recent and less recent results are presented. All of them indicate general relativity theory as a very good description of gravitational phenomena, at least in the studied environment.
\end{abstract}

\section{Introduction}

The general theory of relativity by Albert Einstein is nowadays the most precise description of the gravitational dynamics we have at our disposal. Notwithstanding its precise accounting of gravitational interaction as the effect of curved spacetime on the dynamics of matter and the other fundamental fields, it is challenged by several theoretical ideas, mainly related to the search for a quantum theory of gravitation and to the unification of gravitation itself with the other known fundamental interactions of nature. These issues are ultimately related to the question mark on the smallscale structure of spacetime and to the appearance in the theory of singularities. At the astrophysical and cosmological levels, several unresolved problems may imply a revision of our knowledge of gravitational phenomena. All these issues reflect themselves also on the smaller scale of Solar System, in particular the near-Earth environment, where-thanks to space exploration and more and more advanced experimental techniques-many experimental setups can be conceived and put in place.
Among the ways to test gravitational dynamics one of the simplest is to follow (track) the motion of an object orbiting in the gravitational field produced by another, bigger one (the primary). The orbiting object should be as close as possible to a point mass, in order not to perturb in a significant way the gravitational field of the primary; it should be what is called a test mass. A suitable modellization (analytical or numerical) of this system gives a prediction for the resulting orbit which can be compared with experimental tracking data. Such a scheme is rather general and could be applied to a variety of experimental situations. We describe here a particular such situation, given by the availability around the Earth of objects (satellites) specifically designed to be as much as possible close to the ideal concept of a test mass: the LAGEOS satellites [1]. These, as well as similar ones, have been designed, built and launched for geodetic and geodynamic purposes. In 2012 the LARES satellite has been launched and placed in orbit around Earth. The data from this new laser-ranged satellite, together with those of the LAGEOS, are expected to open the way to still more accurate tests of general relativity; See, for example, [2]. The study of 
their orbital motion, indeed, helps to characterize the fine details of the Earth gravitational field (and therefore of its structure and composition) and to establish and maintain a global reference frame with applications that range from astronomy to navigation (see, e.g., $[3,4]$ ).

The LAGEOS are target for laser pulses sent from ground stations, used to calculate their instantaneous distance (range); the outstanding precision of this tracking technique, named satellite laser ranging (SLR), allows a precise determination of their orbits. This can be done with dedicated procedures and a fine modelling of their dynamics. Along the years, the availability to the scientific community of the ranging data allowed a variety of studies. Many of them, as said above, are related to geodesy and geophysics. At the same time, however, it is possible to exploit the same data to perform fundamental physics tests, by comparing the (measured and reconstructed) orbit with the ones predicted by several, competing, gravitational theories. This very simple objective requires a number of steps to be performed, which will be described in the following. It has to be stressed that, in this quest, to better data better models must follow. This is especially true since the sought for signals often lie several orders of magnitude below the "competitive" signals.

\section{Gravitational Physics Opportunities}

As mentioned above, along the years the LAGEOS satellites turned out to be very good targets to be tracked. They materialize very finely (though not exactly) the ideal concept of a test mass, which has to satisfy the following requirements [5]:

(i) no electric charge,

(ii) gravitational binding energy negligible with respect to rest mass-energy,

(iii) angular momentum negligible,

(iv) sufficiently small to neglect tidal effects.

An ideal test mass follows a purely gravitational orbit (a geodesic in metric theories) and is therefore an appropriate tool to study gravitational phenomena.

2.1. Relativistic Effects on Test Masses around Earth. General relativity, in its weak-field and slow-motion limit, provides an effective description of the gravitational phenomena around Earth. The weak-field condition considers the spacetime curvature so small that the metric can be written as $g_{\mu \nu}=$ $\eta_{\mu \nu}+h_{\mu \nu}$ (Minkowski metric plus a "small" perturbation). The slow-motion condition requires $v \ll c$. Given the relative smallness of the masses at play, as well as that of their speed when compared with that of light, this approximation of the theory is sufficient for the purpose. A formulation of the relevant equations of motion in a geocentric noninertial reference system (nonrotating with respect to the barycentric one) is given in [6], from which we quote the relevant terms. The analyses described here are consistent with this formulation.
A test mass orbiting around Earth is subjected in its motion to three main relativistic effects. The biggest contribution comes from the gravitoelectric curvature of spacetime induced by the Earth mass-energy:

$$
a_{\mathrm{Schw}}=\frac{G m_{\mathrm{E}}}{c^{2} r^{3}}\left[\left(\frac{4 G m_{\mathrm{E}}}{r}-v^{2}\right) r+4(v \cdot r) v\right] .
$$

This is called Einstein or Schwarzschild precession [7]. The satellite, in its motion around Earth, follows its revolution in the spacetime curved by the Sun mass-energy; this (via parallel transport of the normal to the satellite orbit) induces the de Sitter or geodetic precession [8]:

$$
a_{\mathrm{dS}}=2 \Omega \times v \quad \Omega \approx-\frac{3}{2}\left(V_{\mathrm{E}}-V_{\mathrm{S}}\right) \times \frac{G M_{\mathrm{S}} X_{\mathrm{ES}}}{c^{2} R_{\mathrm{ES}}^{3}} .
$$

In general relativity, unlike Newtonian physics, mass-energy currents also cause effects, named gravitomagnetic (see [5]). In particular, Earth intrinsic angular momentum curves spacetime and induces a further effect on the satellite orbit, called Lense-Thirring effect $[9,10]$ (also termed dragging of inertial frames in a more general setting):

$$
a_{\mathrm{LT}}=\frac{2 G m_{\mathrm{E}}}{c^{2} r^{3}}\left[\frac{3}{r^{2}}(r \times v)(r \cdot J)+v \times J\right] .
$$

In the previous expressions, $c$ is the speed of light, $G$ the Newtonian gravitational constant, $m_{\mathrm{E}}$ and $J$ are Earth mass and angular momentum, $r$ and $v$ are the test mass position and velocity in the geocentric frame, $M_{\mathrm{S}}$ is the Sun mass, $V_{\mathrm{E}}$ and $V_{\mathrm{S}}$ are the Earth and Sun geocentric positions, and $X_{\mathrm{ES}}$ is the geocentric Earth-Sun vector, with distance $R_{\mathrm{ES}}$.

Using the methods of celestial mechanics (in particular first-order perturbation theory), the secular effects of relativistic corrections in the satellite Keplerian elements can be evaluated (see, e.g., [11]). In first-order perturbation theory, two kinds of behavior for a given element can arise. The first is a term $\propto \sin t$ or $\cos t$; this is called periodic. The second is a term $\propto t$ (or higher powers); this is called secular, since it tends to accumulate over time. It turns out that the Schwarzschild term is mainly effective on the argument of perigee

$$
\dot{\omega}_{\mathrm{Schw}}=\frac{3\left(G M_{\oplus}\right)^{3 / 2}}{c^{2} a^{5 / 2}\left(1-e^{2}\right)},
$$

the de Sitter one on the longitude of the ascending node

$$
\dot{\Omega}_{\mathrm{dS}}=|\Omega| \cos \varepsilon
$$

(with $\varepsilon$ obliquity of the ecliptic) and the Lense-Thirring one on both node

$$
\dot{\Omega}_{\mathrm{LT}}=\frac{2 G J_{\oplus}}{c^{2} a^{3}\left(1-e^{2}\right)^{3 / 2}}
$$

and perigee

$$
\dot{\omega}_{\mathrm{LT}}=\frac{-6 G J_{\oplus}}{c^{2} a^{3}\left(1-e^{2}\right)^{3 / 2}} \cos I .
$$

Numerical values can be found in Table 1. 
TABLE 1: Rate (mas/yr) and orbital shift (over 14 days) of the different types of secular relativistic precession on LAGEOS and LAGEOS II longitude of ascending node and argument of pericenter, and their sum (1 mas/yr = 1 milli-arc—second per year).

\begin{tabular}{lccr}
\hline & Precession & Rate $(\mathrm{mas} / \mathrm{yr})$ & Shift $(\mathrm{m})$ \\
\hline \multirow{3}{*}{ LAGEOS } & $\Delta \dot{\omega}^{\mathrm{Schw}}$ & 3278.77 & 7.49 \\
& $\Delta \dot{\Omega}^{\mathrm{LT}}$ & 30.88 & $7.46 \times 10^{-2}$ \\
\hline \multirow{3}{*}{ LAGEOS II } & $\Delta \dot{\omega}^{\mathrm{LT}}$ & 32.00 & $7.31 \times 10^{-2}$ \\
& $\Delta \dot{\omega}^{\mathrm{Schw}}$ & 3351.95 & 7.60 \\
& $\Delta \dot{\Omega}^{\mathrm{LT}}$ & 31.48 & $7.14 \times 10^{-2}$ \\
& $\Delta \dot{\omega}^{\mathrm{LT}}$ & -57.00 & $-1.29 \times 10^{-1}$ \\
\hline
\end{tabular}

Are the expected values compatible with the uncertainty associated with tracking data? An estimate of the orbital shift due to each effect can be obtained for nearly circular orbits by $\left.\left.\Delta x\right|_{14 d} \simeq a \Delta \alpha\right|_{14 d} ;$ here $a$ is the semimajor axis of the orbit and $\Delta \alpha$ is the precession (on node or perigee) integrated over the 14 -day estimation period. The values can be seen in the fourth column of Table 1: given a typical SLR Normal Point precision of $\approx 1 \mathrm{~mm}$, we can notice that the Schwarzschild signal is well above the noise, while the gravitomagnetic one is barely above it.

Another important issue is testing for the inverse-square law behaviour of gravitation. On one side, this is useful to better characterize gravitation itself, especially in the short and intermediate range. On another side, possible violations of this behaviour could be related to new interactions between bodies acting at macroscopic distances (new long range interaction (NLRI)). In addition, these NLRIs may be thought of as the residual of a cosmological primordial scalar field related to the inflationary stage (dilaton scenario) [12].

Usually this supplementary interaction is modelled via a Yukawa-type potential added to the Newtonian one, such that, between two bodies of masses $m_{1}$ and $m_{2}$, respectively, at distance $r$ apart

$$
V=-\alpha G_{\infty} \frac{m_{1} m_{2}}{r} e^{-r / \lambda}
$$

Here the Yukawa-type part has a characteristic range $\lambda$ beyond which it becomes negligible, and a relative strength $\alpha$ with respect to the Newtonian part $G_{\infty}$ is the Newtonian constant of gravitation in the limit $r \rightarrow \infty$. The suggestion in the eighties of a possible "fifth force" [13] boosted further research on this (see also [14, 15] for reviews and [16] for recent results).

An adequate observable in order to test for such nonNewtonian behaviour is the pericenter of a binary system. A perturbative analysis of pericenter shift has been performed in [17]. The effect is maximum at a scale comparable with the system semimajor axis; therefore, in the Earth LAGEOS II case, the experiment would be sensitive mainly to an interaction with $\lambda \simeq a$ ( $a$ being the semimajor axis of LAGEOS II orbit). The maximum secular effect is given by

$$
\left\langle\Delta \dot{\omega}^{\mathrm{Yuk}}\right\rangle_{2 \pi} \simeq 8.29 \cdot 10^{11} \alpha(\mathrm{mas} / \mathrm{yr})
$$

and it corresponds to the peak value at a range $\lambda=6082 \mathrm{~km}$, very close to 1 Earth radius.
2.2. Measurement Concept. Among the various techniques used to track satellites, SLR is one of the most precise [18]. It uses the propagation of a collimated laser pulse to measure the instantaneous distance between a station on Earth and a satellite. At the ground station a definite laser pulse is generated and-through a telescope-is sent towards the satellite, where it is reflected back in the same direction by optical elements called cube corner retroreflectors (CCR); it then comes back to the same station, and it is focused by the telescope and detected by a proper sensing device. By precisely measuring the start and stop times of the pulses, it is then possible to recover the instantaneous station-satellite distance (range):

$$
\Delta s=\frac{c \Delta t}{2}
$$

This is of course the basic concept of the measurement. In practice, things are made more complex from having to take into account various phenomena, from the propagation of the pulse in the atmosphere to instrumental biases due to (among other things) laser stability, detector, and timing device. A hint into the complexities of each single measurement can be found in [19]. Laser range observations from the various stations on the globe are collected by the International Laser Ranging Service (ILRS) [18] and are publicly available.

Presently, the two (almost twin) LAGEOS satellites are among the best tracked ones through SLR. LAGEOS, launched by NASA (1976), and LAGEOS II, launched by NASA/ASI (1992), have been designed spherical in shape, with high density and small area-to-mass ratio in order to minimize the effects of the nongravitational perturbations [20]. Their radius is just $30 \mathrm{~cm}$ and their mass about $407 \mathrm{~kg}$. Their aluminum surface is covered with 426 CCRs. LAGEOS has an almost circular orbit, with an eccentricity $e_{\mathrm{I}} \simeq 0.004$, a semimajor axis $a_{\mathrm{I}} \simeq 12270 \mathrm{~km}$ and an inclination over the Earth's equator $i_{\mathrm{I}} \simeq 109.8^{\circ}$. The LAGEOS II corresponding elements are: $e_{\mathrm{II}} \simeq 0.014, a_{\mathrm{II}} \simeq 12162 \mathrm{~km}$ and $i_{\mathrm{II}} \simeq 52.66^{\circ}$. Their aluminum surface is covered with 426 CCRs.

In the analyses described here, a multiarc technique has been employed [21]. The time period considered in the data analysis has been divided into shorter periods, called arcs. For each arc, the tracking data are reduced, resulting in an estimate of the state vector (position and velocity) at the beginning of the arc and of selected parameters for the dynamics. A very precise orbit is therefore obtained for each arc, which can be expressed in terms of Keplerian elements. The arcs have a 1-day overlap, calculating the difference in 
TABLE 2: Magnitude of the main disturbing effects on the LAGEOS II spacecraft (adapted from [20]).

\begin{tabular}{|c|c|c|}
\hline Effect & Estimate & Magnitude $\left(\mathrm{ms}^{-2}\right)$ \\
\hline Earth's monopole & $\frac{G M_{\oplus}}{r^{2}}$ & 2.69 \\
\hline Earth's oblateness & $3 \frac{G M_{\oplus}}{r^{2}}\left(\frac{R_{\oplus}}{r}\right)^{2} \bar{C}_{20}$ & $-1.1 \times 10^{-3}$ \\
\hline Low-order geopotential harmonics & $3 \frac{G M_{\oplus}}{r^{2}}\left(\frac{R_{\oplus}}{r}\right)^{2} \bar{C}_{22}$ & $5.4 \times 10^{-6}$ \\
\hline High-order geopotential harmonics & $19 \frac{G M_{\oplus}}{r^{2}}\left(\frac{R_{\oplus}}{r}\right)^{18} \bar{C}_{18,18}$ & $1.4 \times 10^{-12}$ \\
\hline Moon perturbation & $2 \frac{G M_{\overparen{C}}}{t_{\overparen{C}}^{3}} r$ & $2.2 \times 10^{-12}$ \\
\hline Sun perturbation & $2 \frac{G M_{\odot}}{r_{\odot}^{3}} r$ & $9.6 \times 10^{-13}$ \\
\hline General relativistic correction & $\frac{G M_{\oplus}}{r^{2}} \frac{G M_{\oplus}}{c^{2}} \frac{1}{r}$ & $9.8 \times 10^{-10}$ \\
\hline Atmospheric drag & $\frac{1}{2} C_{D} \frac{A}{M} \rho V^{2}$ & $3.4 \times 10^{-12}$ \\
\hline Solar radiation pressure & $C_{R} \frac{A}{M} \frac{\Phi_{\odot}}{c}$ & $3.2 \times 10^{-9}$ \\
\hline Albedo radiation pressure & $C_{R} \frac{A}{M} \frac{\Phi_{\odot}}{c} A_{\oplus}\left(\frac{R_{\oplus}}{r}\right)^{2}$ & $3.5 \times 10^{-10}$ \\
\hline Thermal emission & $\frac{4}{9} \frac{A}{M} \frac{\Phi_{\odot}}{c} \alpha \frac{\Delta T}{T_{0}}$ & $2.8 \times 10^{-11}$ \\
\hline Dynamic solid tide & $3 k_{2} \frac{G M_{\mathbb{C}}}{r_{\mathbb{C}}}\left(\frac{R_{\oplus}}{r_{\mathbb{C}}}\right)^{2} \frac{R_{\oplus}^{3}}{r^{4}}$ & $3.7 \times 10^{-6}$ \\
\hline Dynamic ocean tide & $\sim 0.1$ of the dynamic solid tide & $3.7 \times 10^{-7}$ \\
\hline
\end{tabular}

elements at the middle of this overlap provides time series of residuals which contain information on the part of dynamics which has not been modelled (or has been mismodelled). The fundamental observable being the range, strictly also the residuals, in their meaning of "observed minus computed", are range. The elements difference method used in these analyses retains the concept for the various Keplerian elements, as shown in [22]. The analysis of the residuals time series allows recovering a posteriori the signature of effects which have not been modelled, as it was purposely done for the relativistic part.

2.3. Analysis Strategy. The tracking data contain the information associated with the satellite dynamics, as well as with the measurement procedure and the observational "constraints" (i.e., station positions, reference frames). This information has to be extracted in some way from the data. The problem is not trivial, considering the relative magnitudes of the effects involved (see Table 2). A direct comparison between the Normal Point precision and the average size of orbit shift due to the relativistic effects shows that these effects could be recovered once the satellite dynamics has been properly modelled (for a description of the models employed see Section 3). The recovery of the information could be done with the least-squares procedure, in which data are fit to a model by a proper estimation of a set of selected parameters.

For the analyses the NASA/GSFC software GEODYN II $[23,24]$ has been used. This software is dedicated to satellite orbit determination and prediction, geodetic parameters estimation, tracking instruments calibration, and many other applications in the field of space geodesy. The software numerically integrates the equations of motion of the satellite using the Cowell's method (a predictor-corrector one, with a fixed time step). The equations of motion for the satellite are integrated in an inertial reference frame, which for GEODYN is the mean equinox and equator of J2000. The orbit determination employs the least-squares solution of the range residuals:

$$
O_{i}-M_{i}=-\sum_{j} \frac{\partial M_{i}}{\partial P_{j}} d P_{j}+d O_{i}
$$

where $O_{i}$ are the range observations, $M_{i}$ are their modelled values, $d P_{j}$ are the corrections to the vector $\mathbf{P}$ of parameters to be estimated, and $d O_{i}$ are the errors associated with each observation. Concerning these errors, the $d \mathrm{O}_{i}$ account for both the contribution from the noise in the observations, as well as for the incompleteness of the mathematical model included in the orbit determination software. The leastsquares algorithm seeks to minimize the residuals $O_{i}-M_{i}$ by adjusting at the same time the state vector at the epoch of arc and the parameters selected for estimation.

A basic choice of the analysis has been to use the residuals in order to recover the relativistic effects. By construction, they provide a measure of the discrepancy between experimental data and models; by purposely not including relativity into the modelling set, the residuals time series is expected to contain signatures of relativity itself. 
TABLE 3: Modelling setup as included in a typical analysis of LAGEOS satellites range data.

\begin{tabular}{lcc}
\hline Model for & Model type & Reference \\
\hline Geopotential (static) & EIGEN-GRACE02S, EGM96 & {$[73,74]$} \\
Geopotential (time-varying, tides) & Ray GOT99.2 & {$[75]$} \\
Geopotential (time-varying, nontidal) & IERS Conventions (2003) & {$[41]$} \\
Third body & JPL DE-403 & {$[52]$} \\
Relativistic corrections ${ }^{\mathrm{a}}$ & Parameterized post-Newtonian & {$[6]$} \\
Direct solar radiation pressure & Cannonball & {$[23]$} \\
Earth albedo & Knocke-Rubincam & {$[76]$} \\
Station positions & ITRF 2000 & {$[77,78]$} \\
Ocean loading & Schernek and GOT99.2 tides \\
Earth Rotation Parameters & IERS EOP C04 & {$[23,75]$} \\
\hline
\end{tabular}

${ }^{a}$ In fact, as explained in the text, these corrections have not been included in the modellization setup used in the analysis.

The basic observable being distance, the residuals are strictly speaking on station-satellite distances. Being interested in effects related to individuals orbital elements, the method outlined in [22] has been employed in order to obtain derived residual time series for the various elements. This is the method that has been employed in the relativistic precessions measurements performed so far [25-33].

The strategy employed here could be considered as "minimal" or "conservative" in the following sense. The precise modelling of the orbits requires complex models, which depend on thousands of parameters (see Section 3). We underline that, while in general geodetic and geophysical problems often the majority of model parameters are estimated, in the analyses only few of them were estimated, namely, those most directly related to the particular orbit of the satellites; the other parameters were selected as consider parameters, that is, ones which are already known with sufficient accuracy from other sources.

This approach considerably simplifies the mathematical structure of the problem being solved, moreover, strongly lowering the chance of estimation biases. In particular, the socalled empirical accelerations have not been included in the set of models fitting the SLR data. These can bias the estimate procedure and corrupt, in particular, the argument of perigee residuals [34].

2.4. LAGEOS Range Data Sets. The basic products of SLR observations are the Fullrate ranges. In the 1980s, a more compact format has been introduced, called Normal Point (NP), which is the one commonly used. A NP is basically an "average" of the Fullrate observations over a defined time period (bin); for the LAGEOS the bin size amounts to $120 \mathrm{~s}$. In the formation of NP for bin $i$, the observation $O_{i}$ nearest to the midpoint of the bin is located, and a fit residual $\mathrm{FR}_{i}$ (a residual from which systematic trends in the predictions have been removed) is calculated. The NP is then calculated as

$$
\mathrm{NP}_{i}=\mathrm{O}_{i}-\mathrm{FR}_{i}+\overline{\mathrm{FR}}_{i}
$$

where $\overline{\mathrm{FR}}_{i}$ denotes the mean value of $\mathrm{FR}_{i}$. The NP so calculated is characterized by the fact that its random error is reduced to that of the mean of the bin. More details can be found in [35].
The precision of the measurements is mainly related with the pulse width, which is usually $\approx 1 \times 10^{-10}$ s down to $3 \times$ $10^{-11} \mathrm{~s}$ for the best laser ranging stations. In the case of the two LAGEOS satellites, the NPs are characterized by a RMS down to a few $\mathrm{mm}$, that corresponds to an accuracy in the orbit reconstruction at a few $\mathrm{cm}$ levels, when using the best dynamical models.

\section{Models}

The procedures for determining the satellite orbit at a level comparable with the quality of tracking data require models not only for satellite dynamics but also for measurement procedure and reference frame transformations. The dynamics of LAGEOS satellites, seen at the level enabled by the accuracy of SLR data, is rather complex. Several gravitational and nongravitational effects are at work; estimates of their magnitude are provided in Table 2 (see [20,36]).

The models included in GEODYN are devoted to describe not only the satellite dynamics, but also the measurement procedure and the reference frame transformations. These models include (i) the geopotential (both in its static and dynamic part), (ii) lunisolar and planetary perturbations, (iii) solar radiation pressure and Earth's albedo, (iv) Rubincam and Yarkovsky-Schach effects (which need the satellite spin-axis orientation in order to be modelled), (v) drag effects, (vi) SLR stations coordinates, (vii) ocean loading, (viii) Earth Orientation Parameters and (ix) measurement procedure. Usually, the models implemented in the code also include the general relativistic corrections in the so-called parameterized post-Newtonian (PPN) formalism [37-40]. In the analyses performed in order to solve for the relativistic secular precessions, such corrections were not included in the setup.

The particular models used for the analyses described here are listed in Table 3. For the relevant part, the Conventions established by the International Earth Rotation and Reference Systems Service (IERS), which constitute the general framework for reference systems related issues and measurement models, have been followed as much as possible. The reference version has been IERS Conventions (2003) [41]. 
3.1. Gravitational Perturbations. The deviations of the Earth's gravitational field from the point mass one, due to the inhomogeneous mass density distribution inside the Earth, are by far the most important source of perturbations in the orbits of LAGEOS satellites. It is customary in geodesy and geophysics to represent the gravitational potential by expanding it in spherical harmonics (real basis):

$$
\begin{aligned}
U(\mathbf{r})= & \frac{G M_{\oplus}}{r} \\
& \times\left[1+\sum_{l=1}^{\infty}\left(\frac{R_{\oplus}}{r}\right)^{l} \sum_{m=0}^{l} \bar{P}_{l m}(\sin \theta)\left(\bar{C}_{l m} \cos (m \phi)+\bar{S}_{l m} \sin (m \phi)\right)\right] .
\end{aligned}
$$

See, for example, $[36,42,43]$. Here $r, \theta$, and $\phi$ represent the polar coordinates of the point at which the potential $U$ is evaluated, $\bar{P}_{l m}$ are the normalized associated Legendre functions, $M_{\oplus}$ is the Earth mass, and $R_{\oplus}$ is the Earth mean equatorial radius. The normalized coefficients $\bar{C}_{l m}$ and $\bar{S}_{l m}$, with $l$ called degree and $m$ order, are function of the mass density distribution, and completely characterize the gravitational potential outside the distribution itself. In practice, the series is truncated at some finite $l_{\max }$ : the model is then sensitive to inhomogeneities at the scale of $\pi R_{\oplus} / l_{\max }$. The lower degree harmonics are related to the choice of the reference frame in which the potential itself is expressed. Of paramount importance are the so-called zonal harmonics, that is, the ones with $m=0$ : they represent the part of the potential with rotational symmetry and play an important role in the error budget of the measurements. Some care must be put in dealing with the permanent tide. In GEODYN, a "tide-free" geopotential is modelled, that is, one in which both the permanent part and the related deformation of the Moon and Sun tidal perturbations have been removed. The $\bar{C}_{2,1}$ and $\bar{S}_{2,1}$ coefficients describe the position of the Earth's figure axis.

The Earth gravitational field, also seen in an "Earth fixed" frame, is not static: it varies in time due to a series of phenomena, from tides to mass transport in the Earth/atmosphere system at various scales. The tidal deformations of the Earth-both solid and ocean-and of its atmosphere are of primary interest for our measurement because of their combined periodic variations in the gravitational attraction of the planet on the satellite [44-49]. In particular, solid tides account for about $90 \%$ of the total response to the Moon and Sun tidal disturbing potential.

A convenient way to describe these deformations is through the so-called Love numbers $\left(k_{2, m}^{f} \simeq 0.30\right.$, where $f$ represents the frequency of the tidal wave), which measure the ratio between the response of the real Earth and the theoretical response of a perfect fluid sphere and are determined with very high accuracy because of their long-term effects on geodetic satellites, as in the case of the two LAGEOS [50, 51]. In particular, in the case of solid tides, the degree $l=2$ terms, that is, those due to the quadrupole tidal potential, are the most important to be considered. Ocean tides are difficult to model because of the greater complexity of the involved phenomena. Indeed, even if ocean tides account for $\approx 10 \%$ only of the total response to the external potentials, their uncertainties are a factor of 10 larger than those of solid tides.

The effect of third-body perturbations has been modelled as well, using the well-established JPL Solar System ephemerides, DE-403 [52]. As discussed in Section 2.1, the relativistic corrections are consistent with the formulation of [6]. In line with the chosen strategy of recovering the relativistic effects a posteriori in the residuals time series, in fact these corrections have been not included in the setup.

3.2. Nongravitational Perturbations. An important part of the satellites dynamics is represented by the effects caused by nongravitational forces. These, of various origin, are caused by the interaction of the satellite body with the near-Earth radiation and particle environment. Such forces are typically surface ones and depend in a complex way on the physical properties of the satellite, as well as on its attitude. Even for very simple satellites as the LAGEOS (spherical in shape, very dense, and passive) these effects are relevant and, especially, very difficult to model. A wide literature is available on the subject; see, for example, [20, 53-55].

The biggest contribution is given by the push of radiation on the satellite surface (radiation pressure), in particular direct visible radiation from the Sun; also reflected visible radiation from Earth (albedo) and infrared radiation emitted from Earth surface are important. They depend on the way this radiation is reflected, diffused, and absorbed by the satellite surface and therefore on the optical properties of this surface.

The most important nongravitational effect is the direct solar radiation pressure. The resultant acceleration, for a body of spherical shape, can be modelled as

$$
\mathbf{a}_{\odot}=-C_{\mathrm{R}} \frac{A}{m} \frac{\Phi_{\odot}}{c}\left(\frac{\left\langle D_{\odot}\right\rangle}{D_{\odot}}\right)^{2} \widehat{\mathbf{s}},
$$

where $A$ is the cross-sectional area of the satellite, $m$ is its mass, $\Phi_{\odot}$ is the solar radiation flux at $1 \mathrm{AU}, c$ is the speed of light, and $C_{R}$ (called radiation coefficient) summarizes the optical properties of the satellite surface. The last squared term is due to the modulation coming from the eccentricity of the Earth orbit around the Sun $\left(D_{\odot}\right.$ is the Earth-Sun distance and $\left\langle D_{\odot}\right\rangle$ its average value) and $\widehat{\mathbf{s}}$ is the Sun unit vector direction. Equation (14) corresponds to the so-called cannonball model for the direct solar radiation pressure from the Sun. This model is rather good for the LAGEOS satellites, provided an estimate is done of the $C_{\mathrm{R}}$ parameter. Evidences have been provided that LAGEOS II optical properties could have been changed since the launch time [56].

More subtle perturbing effects are due to the so-called thermal forces; these are caused by an inhomogeneous temperature distribution of the body (due to its finite thermal inertia), resulting in a thrust force due to emitted radiation. In particular, thermal forces depend on the satellite spin vector, giving different contributions on the orbit as a function of the spin orientation and rate; see, for example, [54]. We have a seasonal-like Yarkovsky-Schach effect in the case of a rapidly spinning satellite, and a diurnal-like Yarkovsky-Schach effect when the fast rotation approximation is no more valid. 


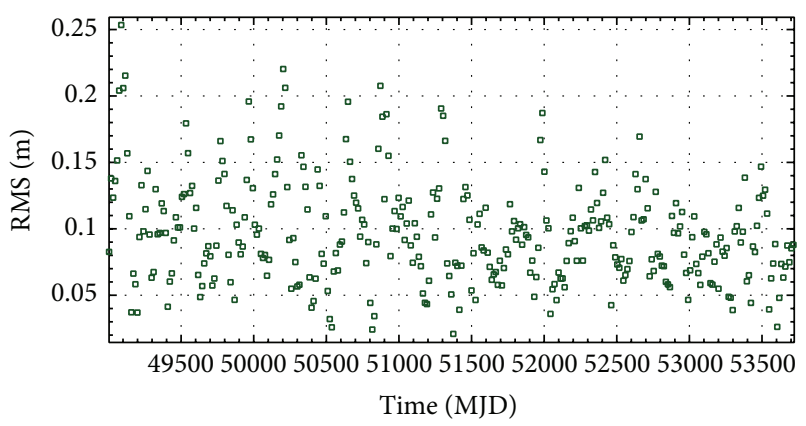

(a)

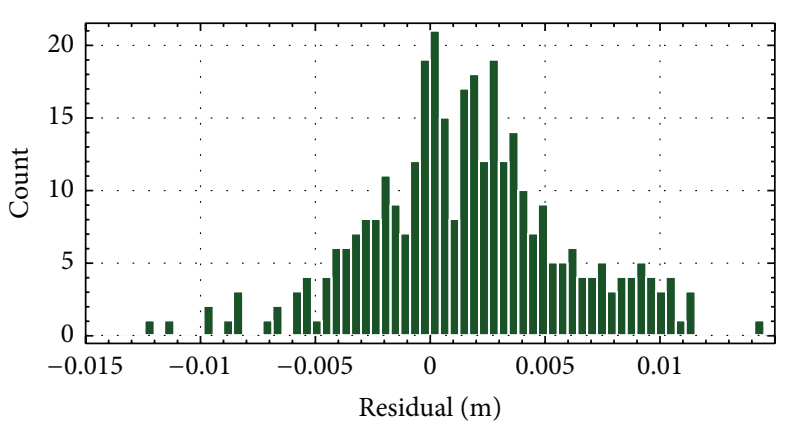

(b)

FIGURE 1: LAGEOS II postfit weighted RMS (a) and residuals in range (b) computed for each of the arcs in which it has been divided the analysed time period.

The Yarkovsky-Rubincam effect [57-59], or Earth-Yarkovsky effect, is related to the infrared radiation emitted by the Earth's surface.

In order to model-as accurately as possible-the perturbing thermal thrust effects, and especially the YarkovskySchach effect, a detailed description of the evolution of the spin-axis is crucial. Several authors have focused on this problem and tried to explain the evolution of the LAGEOS satellites spin-axis, in either an analytical [60-62] or a more empirical approach [63].

3.3. Empirical Accelerations. A modellization piece that is often used in precise orbit determination is given by the socalled empirical accelerations. These are general acceleration terms added to the equations of motion and are aimed at modelling small otherwise unknown effects which may be relevant to the dynamics. They are usually decomposed in the three Gauss directions $\widehat{r}, \widehat{t}, \widehat{w}$ (radial, transverse, and out-ofplane), in the form (for each component)

$$
A(t)=A_{0}+A_{1} \sin M+A_{2} \cos M,
$$

where $M$ represents the satellite mean anomaly, aiming thus at modelling constant or once-per-revolution accelerations. This orbit modelling tool is useful when long wavelengths orbit errors, including secular disturbing effects, need to be removed, as well as for long-period resonances and also nongravitational perturbations that are not included in the software dynamical model. Experience shows that, while they are useful to improve the fit quality, they can easily bias the estimation of other quantities.

We highlight once more that, in order to avoid the orbit corruption, in particular of the satellite argument of pericenter, they have not been used during the data reduction.

\section{Data Reduction}

In the analyses described here more than ten years of LAGEOS and LAGEOS II laser tracking data, provided by ILRS, have been reduced using the GEODYN II software. The selected period has been divided into 15-day arcs, with a 1day overlap. For each of them, the data reduction provides an estimate of the initial conditions (state vector) and of selected parameters. The models employed (see Table 3) enabled a very good fit of the data, as can be seen in the statistics. In particular, in Figure 1 the postfit weighted RMS and a histogram of the residuals in range are shown. These plots are related to runs dedicated to analyze the LAGEOS II perigee behavior (see [31]). The average RMS is somewhat higher than the "ideal" level that could be expected based on data quality: this is due to the fact that in this analysis no relativistic effects were inserted in the modellization set, thereby lowering the overall accuracy. In this way, however, the residuals contain useful information, which is indeed related to relativity itself. This can be seen in the histogram of the residuals in range: their distribution appears close to but is not exactly Gaussian, indicating that some information is still present in the residuals themselves (more information on this can be found in [33]). The same reasoning applies also to the analysis reported in [29]; in that case, however, only the gravitomagnetic contribution was taken out.

\section{Zonal Harmonics Related Uncertainties and Combination Formula for Lense-Thirring Measurements}

Detailed error budget calculations show the importance of the zonal harmonics uncertainties in the overall effectiveness of the analysis procedure in extracting the relativistic signals. This is especially true in the case of Lense-Thirring measurements employing the nodal residuals. In particular, the quadrupole coefficient $\bar{C}_{20}$ has been found to be the major source of uncertainty. Its secular effect on the nodal longitude is given by

$$
\dot{\Omega}_{\text {class }}=\frac{3 \sqrt{5}}{2} n\left(\frac{R}{a}\right)^{2} \frac{\cos I}{\left(1-e^{2}\right)^{2}} \bar{C}_{20} .
$$

See, for example, $[64,65]$ (we use here the normalized coefficients $\bar{C}_{l 0}$ instead of the nonnormalized $C_{l 0}$ or the $J_{l}$; we remember that $J_{2}=-C_{20}=-\sqrt{5} \bar{C}_{20}$ ). Therefore the orbit of the satellite is subject to a classical precession whose value is much higher than the relativistic (gravitomagnetic) one to 
TABLE 4: Values (in mas $\mathrm{yr}^{-1}$ ) of the nodal precession for LAGEOS and LAGEOS II orbits due to relativistic and classical gravitational effects.

\begin{tabular}{lcc}
\hline Effect & LAGEOS & LAGEOS II \\
\hline Lense-Thirring & 30.88 & 31.48 \\
$\bar{C}_{20}$ (EGM96) & $2.702 \times 10^{10}$ & $-4.982 \times 10^{10}$ \\
$\delta \bar{C}_{20}$ (EGM96) & $-3.240 \times 10^{3}$ & $5.975 \times 10^{3}$ \\
$\delta \bar{C}_{20}$ (EIGEN-GRACE02S) & $-2.960 \times 10^{3}$ & $5.458 \times 10^{3}$ \\
\hline
\end{tabular}

be measured; see Table 4 (for the purpose of this work we consider only the quadrupolar part of this classical precession). Of course, what really matters is the unknown part of this precession due to the uncertainty in the accepted value of $\bar{C}_{20}$; its value based on two geopotential models is shown in Table 4 . This precession actually hides the relativistic one and must be handled in some way. To this aim, in [66] a procedure based on the combination of orbital residuals from more than one satellite to get rid of this masking precession has been developed; see also [67].

The simplest case is that of two satellites, along with their two nodal longitudes. Let us consider a single arc of orbit determination; the procedure allows obtaining a residual for each orbital element, in particular for the nodal longitude: $\delta \dot{\Omega}$ (in [22] it is shown that the residuals obtained with their method are in fact rates). If the modellization setup is accurate enough, this residual, that is, the difference between the calculated and the observed value, is mainly function of two quantities, the classical quadrupole precession and the relativistic (Lense-Thirring) one, so that the following equation can be reasonably considered to hold:

$$
\delta \dot{\Omega}=\delta \dot{\Omega}_{\text {class }}+\delta \dot{\Omega}_{\text {rel }}
$$

In writing this equation we neglect higher-degree multipoles and other sources of perturbation for the node. Regarding the two terms on the right-hand side of the previous equation, these can be expressed as follows. Following (16), the classical precession can be written as $\dot{\Omega}_{\text {class }}=N_{20} \bar{C}_{20}$, with $N_{20}$ function of Earth equatorial radius and satellite orbit. Upon the assumption that the biggest uncertainty comes from $\bar{C}_{20}$, one can write

$$
\delta \dot{\Omega}_{\text {class }}=\frac{\partial \dot{\Omega}_{\text {class }}}{\partial \bar{C}_{20}} \delta \bar{C}_{20}=N_{20} \delta \bar{C}_{20} .
$$

In the same way, the relativistic precession can be written as $\dot{\Omega}_{\text {rel }}=\dot{\Omega}_{\mathrm{LT}} \mu$; here $\dot{\Omega}_{\mathrm{LT}}$ comes from (6), while $\mu$ is an empirical parameter measuring the actual value of the relativistic effect $((\mu=0)$ in Newtonian physics, $(\mu=1)$ in general relativity). In fact, in the post-Newtonian framework $\mu=(1+\gamma) / 2$, with $\gamma$ the PPN parameter quantifying how much space curvature is produced by unit rest mass; see [68]. In writing $\dot{\Omega}_{\text {rel }}$ this way, we in fact parameterize the general relativistic prediction with $\mu$ and assume that the error is contained in this empirical parameter (in fact, this equals to admit no a priori knowledge on the amplitude of Lense-Thirring effect), so

$$
\delta \dot{\Omega}_{\mathrm{rel}}=\frac{\partial \dot{\Omega}_{\mathrm{rel}}}{\partial \mu} \delta \mu=\dot{\Omega}_{\mathrm{LT}} \delta \mu .
$$

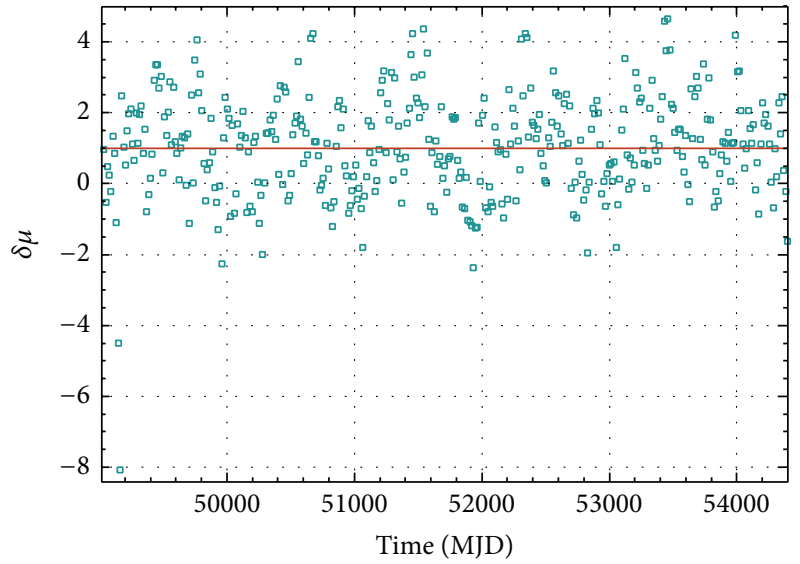

FIGURE 2: Relativistic effect from the combined nodal longitude residuals of LAGEOS and LAGEOS II (squares). The average value is 1.056 , to be compared with the general relativistic prediction value, 1 (continuous line). MJD stands for "Modified Julian Day"; the considered time period for the analysis starts on 1993.

The total uncertainty from (17) can therefore be written as

$$
\delta \dot{\Omega}=N_{20} \delta \bar{C}_{20}+\dot{\Omega}_{\mathrm{LT}} \delta \mu
$$

We see that each residual can be expressed as a function of two uncertainties, $\delta \bar{C}_{20}$ and $\delta \mu$. As such, (20) is not much useful. But adding a further observable (i.e., taking the nodal residuals of two satellites, as LAGEOS and LAGEOS II) one can construct a system of two equations:

$$
\begin{aligned}
& \delta \dot{\Omega}^{\mathrm{I}}=N_{20}^{\mathrm{I}} \delta \overline{\mathrm{C}}_{20}+\dot{\Omega}_{\mathrm{LT}}^{\mathrm{I}} \delta \mu, \\
& \delta \dot{\Omega}^{\mathrm{II}}=N_{20}^{\mathrm{II}} \delta \bar{C}_{20}+\dot{\Omega}_{\mathrm{LT}}^{\mathrm{II}} \delta \mu
\end{aligned}
$$

which can be solved to obtain $\delta \mu$ :

$$
\delta \mu=\frac{N_{20}^{\mathrm{I}} \delta \dot{\Omega}^{\mathrm{II}}-N_{20}^{\mathrm{II}} \delta \dot{\Omega}^{\mathrm{I}}}{N_{20}^{\mathrm{I}} \dot{\Omega}_{\mathrm{LT}}^{\mathrm{II}}-N_{20}^{\mathrm{II}} \dot{\Omega}_{\mathrm{LT}}^{\mathrm{I}}} .
$$

This $\delta \mu$, together with $\delta \bar{C}_{20}$, is just the right one to account for the total residuals $\delta \dot{\Omega}^{\mathrm{I}}$ and $\delta \dot{\Omega}^{\mathrm{II}}$.

It is worth emphasizing two things. First, the solution given by (22) clearly does not contain $\delta \bar{C}_{20}$ and so the expression overcomes the problem of the uncertainty in $\bar{C}_{20}$, both static and time-dependent. Second, $\delta \mu$ as expressed by (22) is related to a single arc of orbit determination, so what one obtains is a time series covering the period of analysis. The outcome can be seen in Figure 2, in which the relativistic 


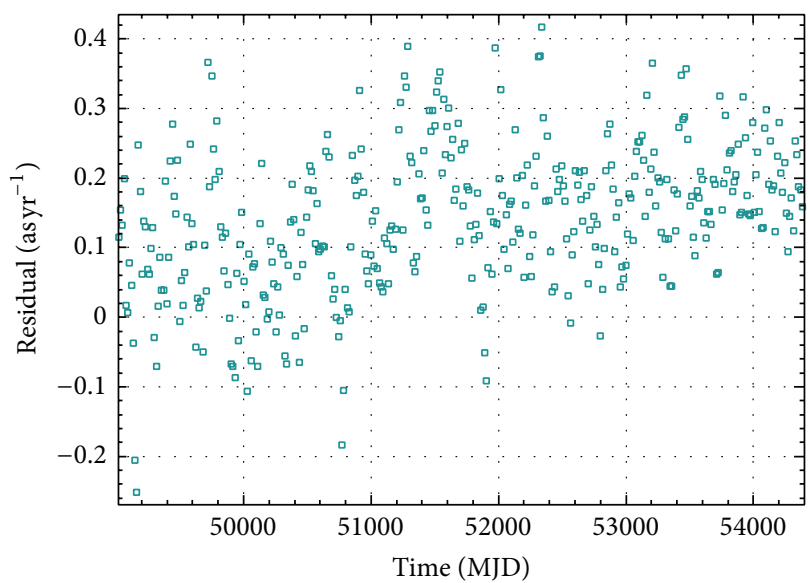

(a)

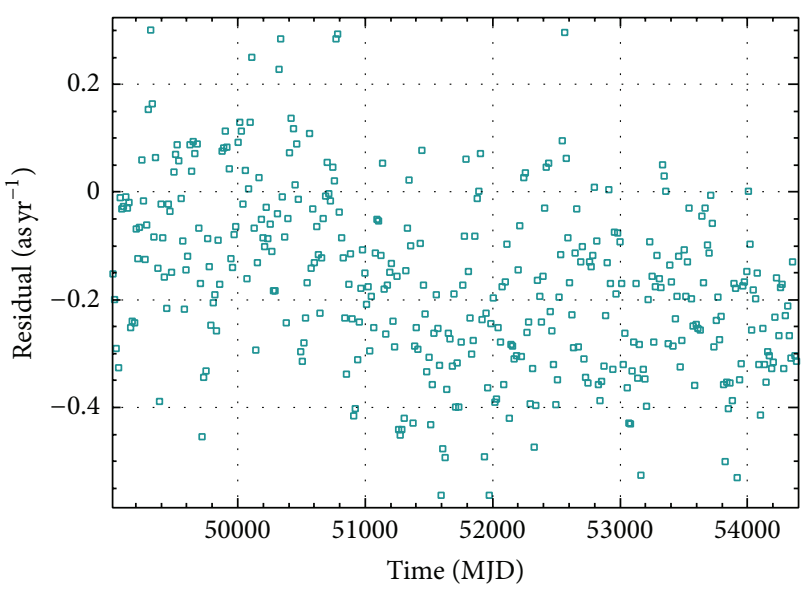

(b)

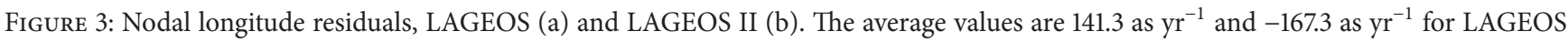
and LAGEOS II, respectively.

parameter $\delta \mu$ from LAGEOS and LAGEOS II combined nodal residuals is plotted as a function of time. Notice the cancellation of the nonseasonal, "anomalous" change in Earth quadrupole (apparent in the nodal longitude residuals; see Figure 3), as reported in $[29,69]$.

\section{A Review of Recent Measurements}

The idea of using laser-ranged satellites in order to test selected predictions of general relativity theory dates back to the 1970s and 1980s. The test of Schwarzschild precession has been discussed in [70]. The measurement of Lense-Thirring effect has been suggested by [71] and proposed by [72]. We review here some recent results which come out from precise orbit determinations of LAGEOS and LAGEOS II satellites. These analyses produced residuals time series of the satellites Keplerian elements in the way discussed in Section 2.2. The expected relativistic signals, both in longitude of ascending node and in argument of perigee, were of a secular type, so they should appear as a secular trend upon time integration of the relevant time series.

In $[28,29,32]$ such an analysis has been performed on LAGEOS and LAGEOS II tracking data, and an accuracy of about $10 \%$ was achieved in the test of the Lense-Thirring effect as predicted by General Relativity. We concentrate here on [29]. Given the limitations due to Earth gravitational quadrupole uncertainty, the node residuals of both satellites have been combined as discussed in Section 5. The corresponding combined time series have been fitted with a secular trend plus a number of periodic terms (in order to account for mismodelling in some perturbations). They report a value of

$$
\mu=0.984 \pm 5 \%-10 \%
$$

using the EIGEN-GRACE02S as geopotential model (the reported value has been obtained fitting the combined residuals with a secular trend plus ten periodic terms). See Figure 4 for the related fit.
In [30] a dedicated analysis has been performed, focused on the LAGEOS II perigee behavior. In that case, the residuals being analyzed were directly those of the Keplerian element: the combination was not necessary, since the overall magnitude of the relativistic effects (Schwarzschild plus LenseThirring) is much bigger. A fit value $\Delta \dot{\omega}_{\text {meas }}=3306.58 \mathrm{mas} / \mathrm{yr}$ for the slope has been reported (see Figure 5). This value can be taken as an estimate of the total relativistic perigee precession, given by

$$
\Delta \dot{\omega}_{\mathrm{rel}} \simeq \varepsilon_{\mathrm{Schw}} \Delta \dot{\omega}_{\mathrm{Schw}}+\varepsilon_{\mathrm{LT}} \Delta \dot{\omega}_{\mathrm{LT}} .
$$

The slope estimate has small variations depending on the number of periodic effects which are fitted together with the linear trend. The following conservative result for the magnitude of the total relativistic effect has been reported, at the post-Newtonian level:

$$
\varepsilon_{\omega}=1+(0.28 \pm 2.14) \times 10^{-3},
$$

where $\varepsilon_{\omega}=1$ in general relativity. Since the dominant contribution in (24) comes from

$$
\varepsilon_{\text {Schw }}=\frac{2+2 \gamma-\beta}{3},
$$

the estimate given by (25) is mainly a measurement of such a combination of $\gamma$ and $\beta$ PPN parameters. A preliminary error budget for the measurement, taking into account the various systematics, estimated the error to be at $2 \%$ level [17]. A complete error budget has been reported in [33]:

$$
\epsilon_{\omega}-1=\left[-0.12 \cdot 10^{-3} \pm 2.10 \cdot 10^{-3}\right] \pm\left[1.74 \cdot 10^{-2}\right],
$$

where in the first square bracket it is shown the result and the statistical error from the best fit and in the second square bracket the error budget due to the gravitational and nongravitational systematic sources of error is represented.

The measured value for the argument of perigee precession can also be used to constrain a non-Newtonian contribution to the satellite dynamics, as discussed in Section 2.1. 


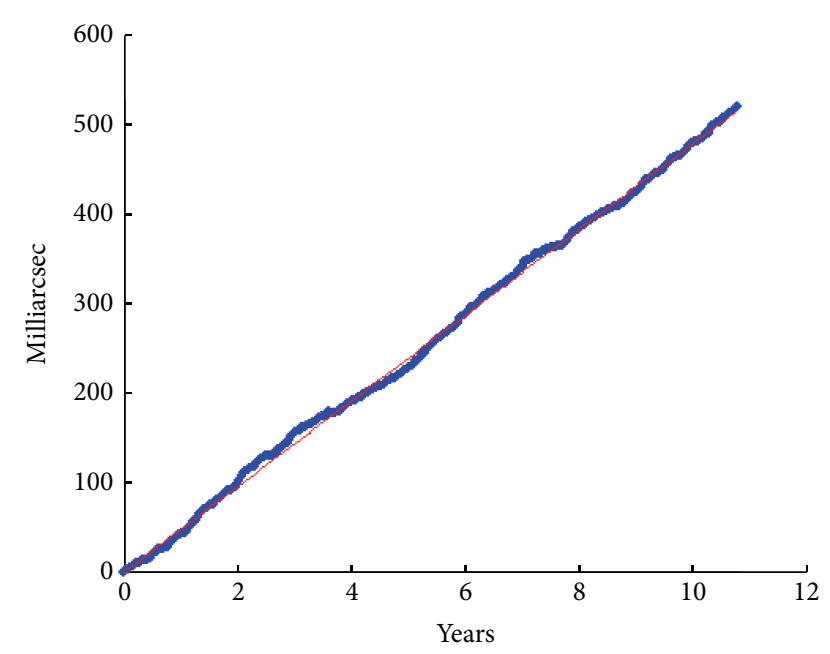

Figure 4: Figure 14 of [29]. A combination of LAGEOS and LAGEOS II node residuals together with their fit with a secular trend plus ten periodic terms is shown.

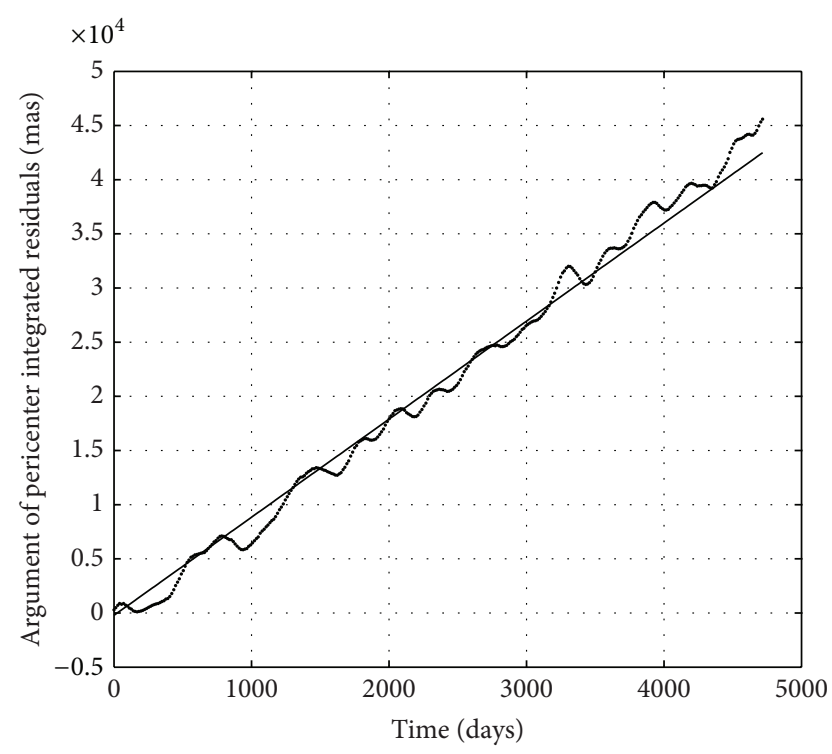

FIGURE 5: Figure 1 of [30]. The perigee residuals are shown together with their fit with a secular trend plus four periodic terms.

Indeed, the absence of such a signal in the residuals time series allows placing a strong constraint to the strength $\alpha$ at $\lambda \simeq a$. In [30] it has been reported the following upper bound:

$$
|\alpha| \simeq|1.0 \pm 8.9| \times 10^{-12},
$$

this result has been improved in [33]:

$$
|\alpha| \simeq|(0.5 \pm 8.0) \pm 69| \cdot 10^{-12} .
$$

These results represent a huge improvement with respect to previous constraints at this scale and are comparable with the Lunar Laser Ranging results.

\section{Conclusions}

There is a great deal of interest in testing the experimental consequences of general relativity, given the many challenges to the theory. Alternative theories, devised to solve at least in part some of these issues, have testable consequences in the weak-field and slow-motion conditions at work in the Solar System, and in particular around Earth. Thanks to advances in experimental techniques, these consequences can be nowadays explored. In particular, laser ranging to geodetic satellites in orbit around Earth offers the possibility of studying with high precision the motion of objects which can be considered very good approximations to a test mass. Their geodetic motion shows some peculiarities with respect to the Newtonian one; in particular, some of the Keplerian elements undergo a precession, also due to gravitomagnetism (the rotating Earth being the source).

Analyses of LAGEOS satellites tracking data, aimed at a precise reconstruction of their orbits, have been discussed. Digging in their dynamics down to the level of the small relativistic effects is made possible not only by the precise laser range data, but also by the accurate modellization of their motion (gravitational and nongravitational parts, reference frames, and measurement models). Some results have been discussed, which provide confirmation of general relativistic predictions (Schwarzschild precession, LenseThirring effect) and rule out to a high degree alternative theories (NLRIs/Yukawa potential). Such investigations have still a great potential of improvement and are being carried on in order to further constrain the space of possible theories.

\section{Conflict of Interests}

The author declares that there is no conflict of interests regarding the publication of this paper.

\section{Acknowledgments}

The author would like to thank David M. Lucchesi (IAPSINAF) and an anonymous referee. The author also acknowledges the ILRS for providing high-quality laser ranging data of the two LAGEOS satellites.

\section{References}

[1] S. C. Cohen, R. W. King, R. Kolenkiewicz, R. D. Rosen, and B. E. Schutz, "LAGEOS scientific results," Journal of Geophysical Research, vol. 90, pp. 9215-9438, 1985.

[2] I. Ciufolini, A. Paolozzi, E. Pavlis et al., "Testing General Relativity and gravitational physics using the LARES satellite," European Physical Journal Plus, vol. 127, article 133, 2012.

[3] G. Xu, Sciences of Geodesy-I: Advances and Future Directions, Springer, Berlin, Germany, 2010.

[4] G. Xu, Sciences of Geodesy-II: Innovations and Future Developments, Springer, Berlin, Germany, 2013.

[5] I. Ciufolini and J. A. Wheeler, Gravitation and Inertia, Princeton University Press, Princeton, NJ, USA, 1995.

[6] C. Huang, J. C. Ries, B. D. Tapley, and M. M. Watkins, "Relativistic effects for near-earth satellite orbit determination," 
Celestial Mechanics and Dynamical Astronomy, vol. 48, no. 2, pp. 167-185, 1990.

[7] A. Einstein, "Die grundlage der allgemeinen relativitätstheorie," Annals of Physics, vol. 354, pp. 769-822, 1916.

[8] W. de Sitter, "On Einstein's theory of gravitation and its astronomical consequences. Second paper," Monthly Notices of the Royal Astronomical Society, vol. 77, no. 155, 184 pages, 1916.

[9] J. Lense and H. Thirring, "Über den Einfluss der Eigenrotation der Zentralkörper auf die Bewegung der Planeten und Monde nach der Einsteinschen GravitationstheoriePhysikalische Zeitschrift," vol. 19, pp. 156-163, 1918.

[10] B. Mashhoon, F. W. Hehl, and D. S. Theiss, "On the gravitational effects of rotating masses: the Thirring-Lense papers," General Relativity and Gravitation, vol. 16, no. 8, pp. 711-750, 1984.

[11] M. H. Soffel, Relativity in Astrometry, Celestial Mechanics and Geodesy, Springer, Berlin, Germany, 1989.

[12] T. Damour, F. Piazza, and G. Veneziano, "Violations of the equivalence principle in a dilaton-runaway scenario," Physical Review D, vol. 66, no. 4, Article ID 046007, 15 pages, 2002.

[13] E. Fischbach, D. Sudarsky, A. Szafer, C. Talmadge, and S. H. Aronson, "Reanalysis of the Eoumltvös experiment," Physical Review Letters, vol. 56, no. 1, pp. 3-6, 1986.

[14] E. Fischbach, G. T. Gillies, D. E. Krause, J. G. Schwan, and C. Talmadge, "Non-Newtonian gravity and new weak forces: an index of measurements and theory," Metrologia, vol. 29, no. 3, pp. 213-260, 1992.

[15] A. S. Goldhaber and M. M. Nieto, "Photon and graviton mass limits," Reviews of Modern Physics, vol. 82, no. 1, pp. 939-979, 2010.

[16] J. H. Gundlach, S. Schlamminger, and T. Wagner, "Laboratory tests of the equivalence principle at the university of washington," Space Science Reviews, vol. 148, no. 1-4, pp. 201-216, 2009.

[17] D. M. Lucchesi, "LAGEOS II perigee shift and Schwarzschild gravitoelectric field," Physics Letters A: General, Atomic and Solid State Physics, vol. 318, no. 3, pp. 234-240, 2003.

[18] M. R. Pearlman, J. J. Degnan, and J. M. Bosworth, "The International Laser Ranging Service," Advances in Space Research, vol. 30, no. 2, pp. 135-143, 2002.

[19] J. J. Degnan, "Satellite laser ranging: current status and future prospects," IEEE Transactions on Geoscience and Remote Sensing, vol. 23, no. 4, pp. 398-413, 1985.

[20] A. Milani, A. M. Nobili, and P. Farinella, Non-Gravitational Perturbations and Satellite Geodesy, Adam Hilger, Bristol, UK, 1987.

[21] A. Milani and G. F. Gronchi, Theory of Orbit Determination, Cambridge University Press, Cambridge, UK, 2010.

[22] D. M. Lucchesi and G. Balmino, "The LAGEOS satellites orbital residuals determination and the Lense-Thirring effect measurement," Planetary and Space Science, vol. 54, no. 6, pp. 581-593, 2006.

[23] D. E. Pavlis et al., GEODYN II Operations Manual. NASA GSFC, 1998.

[24] B. Putney, R. Kolenkiewicz, D. Smith, P. Dunn, and M. H. Torrence, "Precision orbit determination at the NASA Goddard Space Flight Center," Advances in Space Research, vol. 10, no. 3-4, pp. 197-203, 1990.

[25] I. Ciufolini, D. Lucchesi, F. Vespe, and A. Mandiello, "Measurement of dragging of inertial frames and gravitomagnetic field using laser-ranged satellites," Il Nuovo Cimento A, vol. 109, no. 5, pp. 575-590, 1996.
[26] I. Ciufolini, D. Lucchesi, F. Vespe, and F. Chieppa, "Measurement of gravitomagnetism," Europhysics Letters, vol. 39, no. 4, pp. 359-364, 1997.

[27] I. Ciufolini, E. Pavlis, F. Chieppa, E. Fernandes-Vieira, and J. Pérez-Mercader, "Test of general relativity and measurement of the lense-thirring effect with two earth satellites," Science, vol. 279, no. 5359, pp. 2100-2103, 1998.

[28] I. Ciufolini and E. C. Pavlis, "A confirmation of the general relativistic prediction of the Lens-Thirring effect," Nature, vol. 431, no. 7011, pp. 958-960, 2004.

[29] I. Ciufolini, E. C. Pavlis, and R. Peron, "Determination of frame-dragging using Earth gravity models from CHAMP and GRACE," New Astronomy, vol. 11, no. 8, pp. 527-550, 2006.

[30] D. M. Lucchesi and R. Peron, "Accurate measurement in the field of the earth of the general-relativistic precession of the LAGEOS II pericenter and new constraints on non-newtonian gravity," Physical Review Letters, vol. 105, no. 23, Article ID 231103, 2010.

[31] R. Peron, "Einstein is still right: New tests of gravitational dynamics in the field of the Earth," Nuovo Cimento C, vol. 36, pp. 125-134, 2013.

[32] I. Ciufolini, A. Paolozzi, R. Koenig et al., "Fundamental physics and general relativity with the LARES and LAGEOS satellites," Nuclear Physics B-Proceedings Supplements, vol. 243-244, pp. 180-193, 2013.

[33] D. M. Lucchesi and R. Peron, "LAGEOS II pericenter general relativistic precession (1993-2005): error budget and constraints in gravitational physics," Physical Review D, vol. 89, no. 8, Article ID 082002, 2014.

[34] D. M. Lucchesi, “The LAGEOS satellites orbital residuals determination and the way to extract gravitational and nongravitational unmodeled perturbing effects," Advances in Space Research, vol. 39, no. 10, pp. 1559-1575, 2007.

[35] A. T. Sinclair, Data Screening and Normal Point FormationRe-Statement of Herstmonceux Normal Point Recommendation, 1997, http://ilrs.gsfc.nasa.gov/products_formats_procedures/normal_point/np_algo.html.

[36] O. Montenbruck and E. Gill, Satellite Orbits. Models, Methods and Applications, Springer, Berlin, Germany, 2000.

[37] K. Nordtvedt, "Equivalence principle for massive bodies. II. Theory," Physical Review, vol. 169, no. 5, pp. 1017-1025, 1968.

[38] C. M. Will, "Theoretical frameworks for testing relativistic gravity. II. Parametrized post-Newtonian hydrodynamics, and the Nordtvedt effect," The Astrophysical Journal, vol. 163, pp. 611-628, 1971.

[39] C. M. Will and K. Nordtvedt, Jr., "Conservation laws and preferred frames in relativistic gravity. I. Preferred-frame theories and an extended PPN formalism," The Astrophysical Journal, vol. 177, pp. 757-774, 1972.

[40] K. Nordtvedt, Jr. and C. M. Will, "Conservation laws and preferred frames in relativistic gravity. II. Experimental evidence to rule out preferred-frame theories of gravity," The Astrophysical Journal, vol. 177, pp. 775-792, 1972.

[41] D. D. McCarthy and G. Petit, "IERS Conventions (2003)," IERS Technical Note 32, IERS, 2004.

[42] B. Bertotti, P. Farinella, and D. Vokrouhlický, "Physics of the Solar System. Dynamics and Evolution, Space Physics, and Spacetime Structure," in Astrophysics and Space Science Library, vol. 293, Kluwer Academic Publishers, Dordrecht, The Netherlands, 2003. 
[43] B. Hofmann-Wellenhof and H. Moritz, Physical Geodesy, Springer, Berlin, Germany, 2006.

[44] P. Melchior, The Tides of the Planet Earth, Pergamon Press, New York, NY, USA, 1981.

[45] K. Lambeck, "Tidal dissipation in the oceans: astronomical, geophysical and oceanographic consequences," Royal Society of London Philosophical Transactions Series A, vol. 287, pp. 545-594, 1977.

[46] Schwiderski, E. W. : Ocean tides, and part i:, "Global ocean tidal equations," Marine Geodesy, vol. 3, no. 1-4, pp. 161-217, 1980.

[47] E. W. Schwiderski, "Ocean tides, part ii: a hydrodynamical interpolation model," Marine Geodesy, vol. 3, no. 1-4, pp. 219-255, 1980.

[48] J. M. Wahr, "A normal mode expansion for the forced response of a rotating Earth," Geophysical Journal, Royal Astronomical Society, vol. 64, no. 3, pp. 651-675, 1981.

[49] J. M. Wahr, "Body tides on an elliptical, rotating, elastic and oceanless earth," Geophysical Journal, Royal Astronomical Society, vol. 64, pp. 677-703, 1981.

[50] M. K. Cheng, C. K. Shum, and B. D. Tapley, "Determination of long-term changes in the Earth's gravity field from satellite laser ranging observations," Journal of Geophysical Research: Solid Earth, vol. 102, no. 10, pp. 22377-22390, 1997.

[51] B. Wu, P. Bibo, Y. Zhu, and H. Hsu, "Determination of love numbers using satellite laser ranging," The Geodetic Society of Japan, vol. 47, pp. 174-180, 2001.

[52] E. M. Standish, X. X. Newhall, J. G. Williams, and W. M. Folkner, "JPL Planetary and Lunar Ephemerides, DE403/LE403," Tech. Rep. 314. 10-127, JPL IOM, 1995.

[53] D. M. Lucchesi, "Reassessment of the error modelling of nongravitational perturbations on LAGEOS II and their impact in the Lense-Thirring determination. Part I," Planetary and Space Science, vol. 49, no. 5, pp. 447-463, 2001.

[54] D. M. Lucchesi, "Reassessment of the error modelling of nongravitational perturbations on LAGEOS II and their impact in the Lense-Thirring derivation. Part II," Planetary and Space Science, vol. 50, no. 10-11, pp. 1067-1100, 2002.

[55] J. I. Andrés de la Fuente, [Ph.D. thesis], Delft University, 2007.

[56] D. M. Lucchesi, I. Ciufolini, J. I. Andrés et al., "LAGEOS II perigee rate and eccentricity vector excitations residuals and the Yarkovsky-Schach effect," Planetary and Space Science, vol. 52, no. 8, pp. 699-710, 2004.

[57] D. P. Rubincam, "LAGEOS orbit decay due to infrared radiation from earth," Journal of Geophysical Research, vol. 92, pp. 1287-1294, 1987.

[58] D. P. Rubincam, "Yarkovsky thermal drag on LAGEOS," Journal of Geophysical Research, vol. 93, no. 1113805, p. 13810, 1988.

[59] D. P. Rubincam, "Drag on the LAGEOS satellite," Journal of Geophysical Research, vol. 95, pp. 4881-4886, 1990.

[60] B. Bertotti and L. Iess, "The rotation of LAGEOS," Journal of Geophysical Research, vol. 96, no. 2, pp. 2431-2440, 1991.

[61] S. Habib, D. E. Holz, A. Kheyfets, R. A. Matzner, W. A. Miller, and B. W. Tolman, "Spin dynamics of the LAGEOS satellite in support of a measurement of the Earths gravitomagnetism," Physical Review D, vol. 50, no. 10, pp. 6068-6079, 1994.

[62] S. E. Williams, The Lageos satellite: a comprehensive spin model and analysis [Ph.D. thesis], North Carolina State University, Raleigh, NC, USA, 2002.

[63] J. Ries, R. Eanes, and M. M. Watkins M, "Spin vector influence on LAGEOS ephemeris," presented at the Second Meeting of IAG Special Study Group 2. 130, Baltimore, Md, USA, 1993.
[64] W. M. Kaula, Theory of Satellite Geodesy. Applications of Satellites to Geodesy, Blaisdell, Waltham, Mass, USA, 1966.

[65] L. Iorio, "The impact of the static part of the earth's gravity field on some tests of general relativity with satellite laser ranging," Celestial Mechanics and Dynamical Astronomy, vol. 86, no. 3, pp. 277-294, 2003.

[66] I. Ciufolini, "On a new method to measure the gravitomagnetic field using two orbiting satellites," Il Nuovo Cimento A, vol. 109, no. 12, pp. 1709-1720, 1996.

[67] R. Peron, "On the use of combinations of laser-ranged satellites orbital residuals to test relativistic effects around Earth," Monthly Notices of the Royal Astronomical Society, vol. 432, pp. 2591-2595, 2013.

[68] C. M. Will, "The confrontation between general relativity and experiment," Living Reviews in Relativity, vol. 4, article 4, 2001.

[69] C. M. Cox and B. F. Chao, "Detection of a large-scale mass redistribution in the terrestrial system since 1998," Science, vol. 297, no. 5582, pp. 831-833, 2002.

[70] D. P. Rubincam, "General relativity and satellite orbits: the motion of a test particle in the Schwarzschild metric," Celestial Mechanics, vol. 15, no. 1, pp. 21-33, 1977.

[71] L. Cugusi and E. Proverbio, "Relativistic effects on the motion of earth's artificial satellites," Astronomy and Astrophysics, vol. 69, pp. 321-325, 1978.

[72] I. Ciufolini, "Measurement of the Lense-Thirring drag on high-altitude, laser-ranged artificial satellites," Physical Review Letters, vol. 56, no. 4, pp. 278-281, 1986.

[73] C. Reigber, R. Schmidt, F. Flechtner et al., "An Earth gravity field model complete to degree and order 150 from GRACE: EIGEN-GRACE02S," Journal of Geodynamics, vol. 39, no. 1, pp. $1-10,2005$.

[74] F. G. Lemoine, S. C. Kenyon, J. K. Factor et al., “The development of the joint nasa gsfc and the national imagery and mapping agency (nima) geopotential model egm96," Technical Paper NASA/TP-1998-206861, NASA GSFC, 1998.

[75] R. D. Ray, "A Global Ocean Tide Model From TOPEX/ POSEIDON Altimetry: GOT99. 2," Technical Paper NASA/ TM-1999-209478, Goddard Space Flight Center, Greenbelt, Md, USA, 1999.

[76] D. P. Rubincam, P. Knocke, V. R. Taylor, and S. Blackwell, "Earth anisotropic reflection and the orbit of LAGEOS," Journal of Geophysical Research, vol. 92, pp. 11662-11668, 1987.

[77] Z. Altamimi, P. Sillard, and C. Boucher, "ITRF2000: new release of the International Terrestrial Reference Frame for earth science applications," Journal of Geophysical Research B: Solid Earth, vol. 107, no. 10, pp. 2-19, 2002.

[78] C. Boucher, Z. Altamimi, P. Sillard, and M. Feissel-Vernier, “The ITRF2000," IERS Technical Note 31, IERS, 2004.

[79] "International Earth Rotation Service: EOP Combined Series EOP”, Tech. Rep. C04, IERS. 

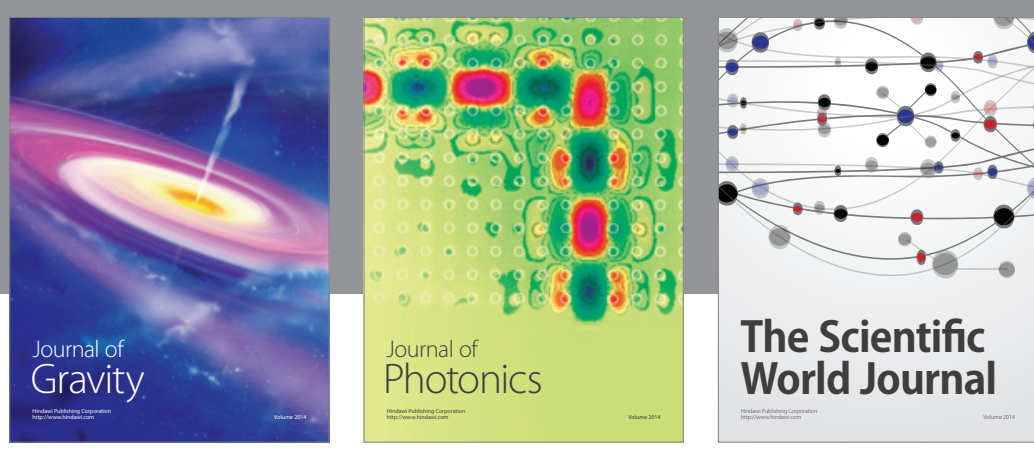

The Scientific World Journal
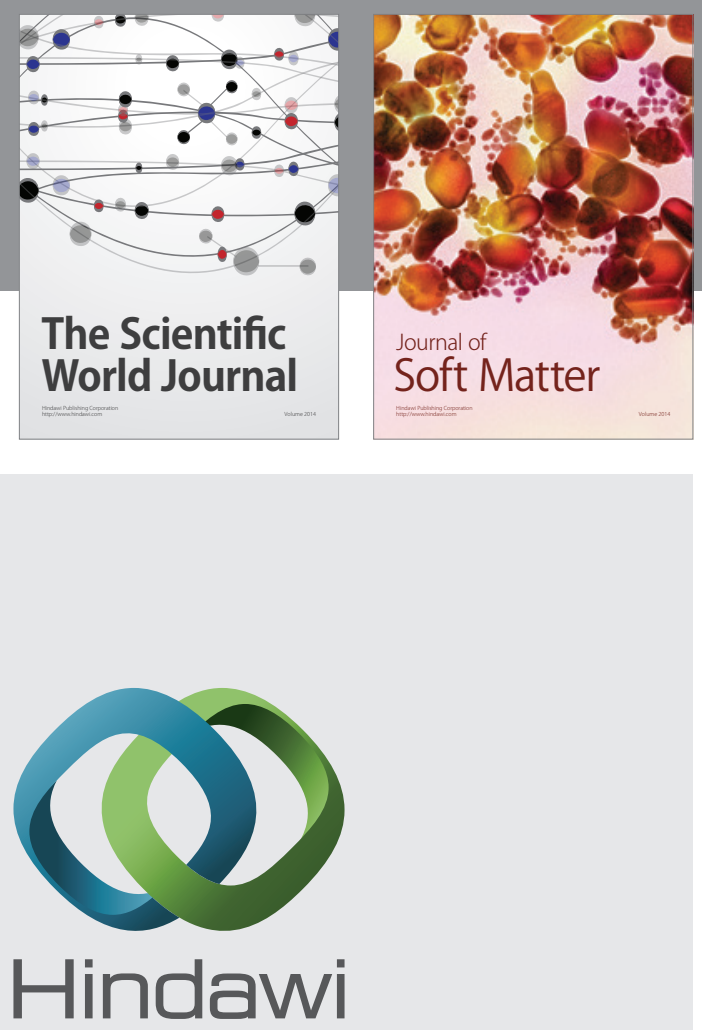

Submit your manuscripts at

http://www.hindawi.com

nternational Journal of

Statistical Mechanics
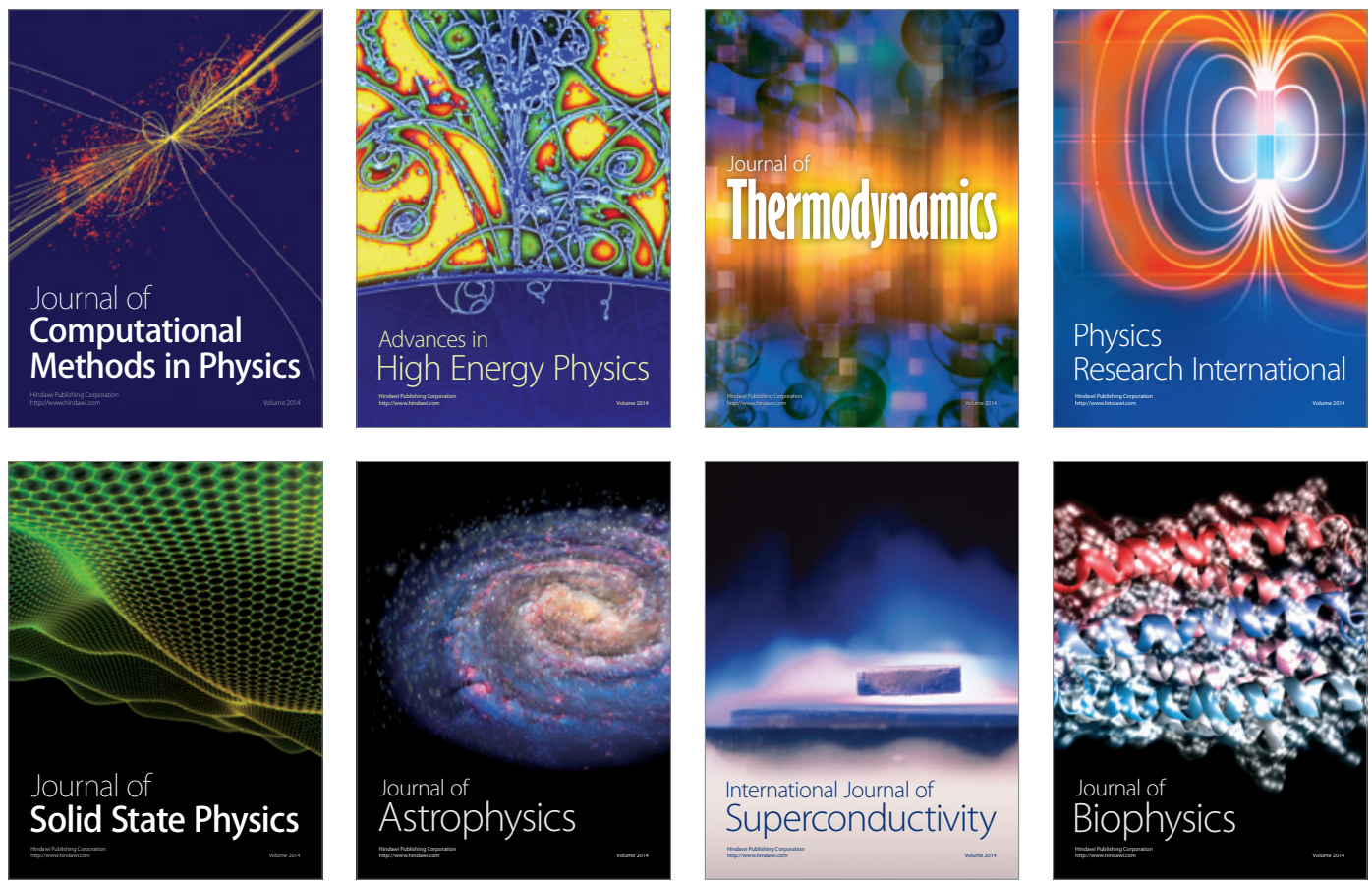
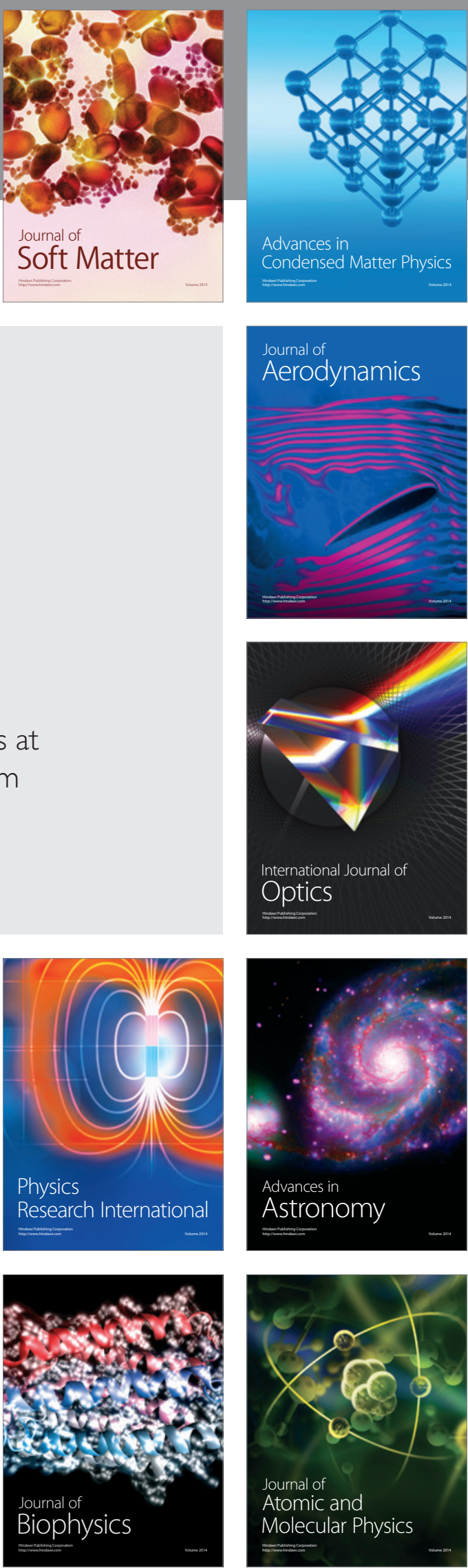